\section{Comportamento alimentar neonatal}

\section{Neonatal feeding behavior}

Sandra Lopes de Souza 1

Raul Manhães de Castro 2

Maria Inês Nogueira 3

1 Instituto de Ciências Biomédicas. Universidade de São Paulo. 2 Departamento de Nutrição. Universidade Federal de Pernambuco. Av. Prof. Moraes Rego, 1235. Cidade Universitária. Recife, PE, Brasil. CEP: 50.670-901

3 Departamento de Anatomia. Instituto de Ciências Biomédicas. Universidade de São Paulo

\begin{abstract}
Feeding behavior suffers intense changes during lactation. Several studies describe the necessary changes in behavior involved in the feeding pattern of newborns. The control of this behavior also depicts physiological adjustment through lactation, changing from a pre-absorptive pattern in earlier stages to a post-absorptive pattern close to weaning. Because of these changes this state is vulnerable to environmental aggressions that could influence the establishment of feeding standards associated to pathologies in adult life.
\end{abstract}

Key words Feeding behavior, Breast feeding, Infant, newborn
Resumo Durante o período de aleitamento, aspectos do comportamento alimentar sofrem intensa transformação. Vários estudos descrevem, do nascimento ao desmame, as modificações nas ações comportamentais necessárias para aquisição do alimento pelo neonato. O controle desse comportamento também apresenta ajustes fisiológicos através da lactação, mudando de um padrão pré-absortivo, em período precoce, para pós-absortivo, próximo ao desmame. Devido a essas transformações, esse período torna-se vulnerável a agressões ambientais que podem influenciar o estabelecimento de padrões alimentares que estarão associados a patologias na vida adulta.

Palavras-chave Amamentação, Conduta na alimentação, Recém-nascido 


\section{Introdução}

A aquisição de matéria-prima essencial e de fontes de energia do meio, no início da vida, é importante para o crescimento bem como para o desenvolvimento físico e psicológico do ser humano. ${ }^{1}$ Para os mamíferos, a nutrição no período pós-natal precoce é representada pelo aleitamento materno. $\mathrm{O}$ ato de amamentar é importante ademais por favorecer o contato materno, tendo efeito calmante e inclusive analgésico sobre o lactente. 2,3 $\mathrm{A}$ amamentação e a interação materno-infantil podem pois modular o estado comportamental da criança ${ }^{2}$ e da mãe, 4 influenciando o desenvolvimento psicológico não só afetivo como de aprendizado. Assim, o estudo do comportamento alimentar neonatal torna-se de extrema relevância, tendo em vista a gama de influências que pode exercer sobre a consolidação de padrões comportamentais.

Nas últimas décadas, vários estudos têm descrito as modificações comportamentais e dos mecanismos de controle da ingestão alimentar no período entre o nascimento e o desmame.5-8 A identificação de tais modificações tem sido obtida principalmente em estudos experimentais. 9 Nessa fase, alguns estímulos ambientais, como o excesso ou a escassez na oferta de alimento, podem consolidar padrões alimentares que estarão eventualmente associados à patologias na vida adulta. 10 A subnutrição no período fetal e/ou neonatal precoce, por exemplo, tem sido apontada como fator de risco para doenças cardiovasculares no adulto. 11

Nessa revisão, será descrita a sequiência de comportamentos do neonato que permite a amamentação, bem como suas características em modelos animais e humanos. Também serão abordados os mecanismos de controle neonatal da ingestão alimentar, particularmente os aspectos que os diferenciam do controle no adulto. Para finalizar, serão descritas as possíveis relações entre perturbações do padrão alimentar no início da vida e o desencadeamento de eventuais mudanças estruturais e funcionais permanentes. Estas poderiam ser responsáveis pelo desenvolvimento de patologias como as coronariopatias, a hipertensão arterial e a obesidade na vida adulta.

\section{Características do comportamento alimentar neonatal}

Há inúmeras pesquisas utilizando o rato como modelo experimental,12-14 porém estudos da ontogênese do comportamento alimentar em humanos ainda são limitados. ${ }^{7}$ No rato, o período de lactação corres- ponde aos 21 primeiros dias de vida.

Para a aquisição do leite materno, o lactente mamífero (em quase todas as espécies) apresenta um fenótipo comportamental característico, respeitando uma seqüência temporal de condutas que varia segundo as espécies. Expressos em diferentes animais, exaustivamente estudados experimentalmente e, inclusive, já descritos na literatura, 14,15 os comportamentos do ato de se aleitar são organizados nas seguintes etapas:

\section{Procura do mamilo materno}

Essa etapa caracteriza-se, no rato, principalmente por movimentos de membros anteriores, promovendo rotação e direcionamento do neonato para a região ventral materna. ${ }^{9}$ Com o desenvolvimento locomotor e abertura dos olhos há atenuação do processo de procura com rápida orientação para $o$ mamilo. 15

\section{Fixação ao mamilo}

Ao aproximar-se da mãe, o neonato orienta-se por fontes de calor para a fixação ao mamilo, apresentando movimentos rítmicos de cabeça, boca e língua. $16 \mathrm{O}$ olfato parece ser crítico nesse processo. No rato, quando o filhote é privado desse sentido, não inicia a sucção e após alguns dias morre de inanição. 17 Essa função do olfato no aleitamento parece ser resultado do aprendizado. Após o parto, as ratas distribuem o líquido amniótico na superfície dos mamilos, o que favorece o reconhecimento destes pelos filhotes e a primeira fixação. $18 \mathrm{Em}$ recém-nascidos humanos, foi demonstrada predileção aos mamilos das mães, preferencialmente antes da remoção de substâncias odoríferas, em contraposição aos de outra lactante. ${ }^{19}$

\section{Ingestão de leite}

Apresenta duração, freqüência e quantidade modificadas com a idade do neonato. Em ratos, esses três fatores sofrem redução gradual em direção ao desmame. 15,20 Em humanos, nos primeiros quatro meses de vida, foi observado declínio na duração e frequiência de sucção, não havendo alteração na ingestão total de leite. 21

\section{Desligamento do mamilo}

Ratos lactentes com cinco dias de idade apresentam certo esforço para desligarem-se do mamilo materno, ficando muitas vezes ofegantes. No décimo dia, 
empurram o mamilo após o leite ter preenchido suas bocas, não sendo mais observadas alterações respiratórias. Próximo ao desmame (vigésimo dia de vida), os filhotes finalizam a sucção desligando-se ativamente do mamilo materno.

\section{Comportamentos após a sucção}

Ratos neonatos sonolentos deitam próximos à região do mamilo materno quando muito jovens. Ao alcançarem o $15^{\circ}$ dia de vida, metade dos filhotes de uma ninhada apresenta comportamentos característicos do adulto, como o de limpeza e o exploratório. No entanto, somente ao $20^{\circ}$ dia de vida, é observada em toda a ninhada uma seqüência de comportamentos característica do adulto: ingestão alimentar, comportamentos de exploração e de limpeza, sono (não dormem próximo ao ventre materno). Essa é a seqüência comportamental de saciedade presente ao término da ingestão alimentar. 22

\section{Controle do comportamento alimentar neonatal}

A regulação do comportamento alimentar ocorre, segundo York, 23 por interação complexa entre mecanismos de controle periféricos e centrais. Por conseguinte, o comportamento alimentar é influenciado por vários fatores, e entre eles estão os determinantes ambientais, os orgânicos e os fisiológicos. 24 As alterações fisiológicas associadas à alimentação ou jejum geram sinais de fome ou saciedade. Através desses sinais, o animal estará hábil a determinar quando e como o alimento deverá ser ingerido para manter sua sobrevivência. 24 A contração e a distensão do estômago são os principais mecanismos periféricos pré-prandiais que contribuem para a sensação de fome e saciedade, respectivamente. ${ }^{25}$ Após a absorção dos nutrientes, fatores pós-prandiais como hormônios (colecistocinina, insulina), aumento da temperatura corporal e nutrientes (principalmente a glicose) contribuem para o final da alimentação. 26

Contudo, no neonato alguns dos mecanismos envolvidos no controle do comportamento alimentar são imaturos, apresentando algumas peculiaridades. Assim, estudos experimentais, têm acompanhado a ontogênese desses mecanismos. O controle do comportamento alimentar parece mudar de um padrão pré-prandial, durante períodos precoces do aleitamento, para pós-prandial, próximo ao desmame. 27 Ratos neonatos não ajustam a ingestão alimentar em resposta a sinais fisiológicos pós-prandiais, como o nível de glicose sanguínea. 28 Esse mecanismo de controle parece não ser ativo nessa idade. ${ }^{12}$ Assim, uma carga alimentar calórica com $20 \%$ de glicose reduz a ingestão na mesma proporção que uma não calórica, com $20 \%$ de maltose, substância não metabolizada no organismo. ${ }^{28}$ Isso evidencia o papel principal dos sinais fisiológicos pré-prandiais, como a distensão do trato gastrointestinal, na regulação da ingestão até o $12^{\circ}$ dia de vida. Outro fator relevante no controle da ingestão em neonatos é a habituação oral. Em resposta a uma série de curtas infusões orais, os filhotes apresentam reduções progressivas nos movimentos de mandíbula e da língua. ${ }^{29}$ Os animais podem habituar-se aos estímulos próprios do alimento e como resultado dessa habituação cessam a ingestão. Este é um processo primário que atua sinalizando o término dos episódios ingestivos produzindo saciedade. 30 Em recém-natos descerebrados desta espécie, a habituação oral e sua integração com sinais de enchimento gástrico continuam atuantes, sugerindo que baixo nível neural é suficiente para manter esse mecanismo. 30

$\mathrm{O}$ grande volume de leite ingerido até o $10^{\circ}$ dia de vida, em ratos, é um indicador da imaturidade do controle da ingestão alimentar dos neonatos. Quando há oferta ilimitada de leite, os filhotes ingerem o volume máximo da capacidade do trato gastrointestinal. 5 Durante o período de aleitamento, o tamanho das refeições aumenta enquanto que sua freqüência diária diminui. $27 \mathrm{Em}$ neonatos humanos, dados semelhantes foram observados, com aumento na quantidade de leite ingerido por mamada e no período entre as mamadas. 6 Em meses sucessivos de aleitamento materno exclusivo, o número total de mamadas e a duração por mamada diminuem. ${ }^{21}$ Possivelmente a criança torna-se mais eficiente em esvaziar a mama materna, ou a necessidade de sucção não nutritiva (sem ingestão de leite) pode diminuir com o desenvolvimento. ${ }^{21} \mathrm{O}$ tamanho aumentado da refeição e intervalo entre as mamadas pode representar alterações preliminares que ocorrem antes do desmame. ${ }^{7}$ Nesse último período, ratos ingerem quantidades menores de leite indicando maturação do controle da ingestão alimentar. ${ }^{5}$ Os sinais fisiológicos pós-prandiais são eficientes em finalizar a refeição, de modo que, há aumento da ingestão em resposta a solução não calórica, mas não há para a calórica. ${ }^{13}$

A ingestão de alimentos sólidos pode iniciar-se a partir do $12^{\circ}$ dia de vida em ratos. Nesse mamífero, parece provável que os filhotes sejam estimulados a comer alimento sólido quando a mãe também o faz. ${ }^{31}$ A presença da mãe influencia o tipo de alimento selecionado 32 e o tempo inicial de alimentação. 33 Em neonatos humanos o aleitamento materno exclusivo é recomendado até seis meses de idade. 34 A par- 
tir dessa idade a introdução de alimentos sólidos inicia-se, tornando esta fase muito importante para consolidação dos hábitos alimentares.

\section{Comportamento alimentar neonatal: repercussões na vida adulta}

O hipotálamo é uma das principais estruturas do sistema nervoso central envolvida na regulação do comportamento alimentar. O período crítico de diferenciação e maturação dessa estrutura é pós-natal. 35 Durante esse período o hipotálamo é vulnerável à influências ambientais que podem modificar tanto a sua estrutura quanto a sua função, o que pode ser base para o desencadeamento de patologias na vida adulta. 36

A obesidade, além de se desenvolver como consequiência de anormalidades genéticas, também pode ser induzida por vários fatores ambientais, especialmente quando incidem na vida precoce. 37 Existe um grande número de adultos obesos que já apresentam obesidade desde a infância, apoiando a hipótese de que essa patologia tenha início ainda nessa fase precoce da vida. 38 Em alguns estudos com ratos, observou-se que a manipulação da quantidade de alimento disponível para os filhotes durante o período de aleitamento altera de forma persistente o comportamento alimentar na vida adulta. ${ }^{39}$ Assim, quando há grande quantidade disponível de leite, ocorre persistência de hiperfagia acompanhada por sobrepeso. 39

A leptina é uma proteína produzida pelo tecido adiposo que promove redução na ingestão alimentar e aumento do gasto energético.40,41 Ratos adultos supernutridos durante o período de aleitamento apresentam redução na ação da leptina sobre o hipotálamo. Essa resistência adquirida contribui para o desenvolvimento de hiperfagia e sobrepeso na vida adulta. 40 Associado a essa alteração observou-se hiperinsulinismo, fator que também contribui para o sobrepeso. ${ }^{36} \mathrm{~A}$ insulina pode induzir malformação dos núcleos hipotalâmicos levando a alterações funcionais permanentes relacionadas com a expressão e responsividade de receptores para a insulina. 36 Outro fator observado foi o aumento na responsividade de neurônios hipotalâmicos para a dopamina. 42 Esse neurotransmissor inibe o centro hipotalâmico que promove a saciedade, contribuindo assim, para a hiperfagia persistente. 43 Pode-se concluir que a maior disponibilidade de alimento na vida pós-natal precoce pode influenciar o desenvolvimento dos mecanismos hipotalâmicos reguladores do comportamento alimentar, induzindo estabilização da eleva- da ingestão de alimentos por toda a vida. ${ }^{8}$

Em neonatos humanos, a utilização de fórmulas artificiais comparadas ao leite materno, pode levar a maior ingestão alimentar e conseqüentemente predispor à obesidade. $44 \mathrm{~A}$ utilização de fórmulas pode induzir hipercolesterolemia no adulto, estando associada ao desencadeamento de doença arterosclerótica. 45 Por outro lado, crianças amamentadas com leite materno apresentam menores níveis de colesterol na vida adulta. ${ }^{46}$ Esse resultado sugere que a ingestão de leite materno pode programar o metabolismo de gordura e reduzir subseqüentemente o risco de doenças cardiovasculares. 45

Por outro lado, a deficiência nutricional no início da vida também constitui fator importante na consolidação dos mecanismos reguladores do comportamento alimentar, promovendo alterações persistentes. A desnutrição é capaz de alterar os eventos morfogenéticos com conseqüências deletérias para o desenvolvimento e aquisição de padrões fisiológicos maduros. 47 Assim, alterações no número de células cerebrais e adiposas que ocorrem na desnutrição em período precoce, podem alterar mecanismos periféricos ou cerebrais que estão envolvidos no controle do ato alimentar. Além disso, pode produzir marcadas alterações químicas e funcionais no cérebro. ${ }^{48}$ Uma das estruturas encefálicas particularmente vulnerável a essa carência no início da vida é o hipotálamo. Desnutrição protéica, nesse período, promove desorganização de centros hipotalâmicos reguladores do peso corporal e do metabolismo. 49

Desnutrição imposta durante o período de aleitamento promove maior tempo gasto em sucção, em prejuízo da expressão de alguns comportamentos maternos como o descanso e a alimentação. A modificação desses comportamentos maternos ocorre possivelmente na tentativa de garantir crescimento e desenvolvimento normais dos filhotes, pois o maior tempo gasto no ninho aumenta a disponibilidade de leite. 20,50

Ratos desnutridos no período de aleitamento apresentam aumento da ingestão alimentar e hídrica ${ }^{51}$ e não respondem ao efeito anoréxico da fluoxetina (um inibidor seletivo de recaptação de serotonina) na vida adulta. 52 Pode-se sugerir que a desnutrição precoce promove hipo-responsividade de receptores serotoninérgicos de modo permanente, o que pode alterar o comportamento alimentar adulto. 52

A desnutrição na infância, seguida de recuperação nutricional, condiciona maior morbi-mortalidade por doença cardíaca isquêmica na vida adulta. 53 No estudo de Fall et al. 54 foi verificado que a prevalência de doença coronariana decresce de $27 \%$ naqueles que pesavam $8,2 \mathrm{~kg}$ ou menos com um ano de idade 
para $9 \%$ naqueles com peso superior a $11,8 \mathrm{~kg}$. O processo arterosclerótico pode ter início na infância evoluindo durante a vida. A arterioesclerose coronariana é a principal causa de doença cardíaca isquêmica. Esta apresenta como um dos fatores de risco o baixo peso ao nascer ou no primeiro ano de vida, o que reflete má nutrição. 38

\section{Conclusões}

É possível identificar as distintas fases da expressão de comportamentos que permitem ao neonato fixar- se ao mamilo materno. Essas fases estão associadas à maturação do sistema nervoso central, que se desenvolve de forma considerável durante o aleitamento. Assim, na fase precoce do comportamento alimentar, o controle fisiológico é exercido, particularmente, por estímulos pré-absortivos como a distensão do trato gastrointestinal. Próximo ao desmame, estímulos pós-absortivos assumem o controle desse comportamento. O conhecimento dessas fases e suas características constitui ferramenta importante para correlacionar agressões que incidem sobre o aleitamento e patologias na vida adulta, particularmente os distúrbios alimentares.

\section{Referências}

1. Lipsitt PL, Crook C, Booth CA. The transitional infant: behavioral development and feeding. Am J Clin Nutr 1985; 41: 485-96.

2. Smotherman WP, Robinson SR. Milk as the proximal mechanism for behavioral change in the newborn. Acta Paediatric 1994; 397: 64-70.

3. Gray L, Watt L, Blass EM. Skin-to-skin contact is analgesic in healthy newborns. Pediatrics 2000; 105: 14-9.

4 Kinsley CH, Madonia L, Gifford GW, Tureski K, Griffen GR, Lowry C, Williams J, Collins J, McLearie H, Lambert KG. Motherhood improves learning and memory. Nature 1999; 402: 137-8.

5. Houpt KA, Epstein AN. Ontogeny of controls food intake in the rat: GI fill and glucoprivation. Am J Physiol 1973; 225: 58-66.

6. Cramer CP, Blass EM. Mechanisms of control of milk intake in suckling rats. Am J Physiol Regul Integr Comp Physiol 1983; 245: R154-9.

7. Matheny RJ, Birch LL, Picciano MF. Control of intake by human-milk-fed infants: relationships between feeding size end interval. Dev Psychobiol 1990; 23: 511-8.

8. Zippel U, Heidel E, Plagemann A, Davidowa H. Action of CCK and 5-HT on lateral hypothalamic neurons depends on early postnatal nutrition. Nutr Neurosci 2001; 4: 14352.

9. Blass EM, Teicher MH. Suckling. Science 1980; 210: 15-22.

10. Kramer MS, Barr RG, Leduc DG, Boisjoly C, Pless IB. Infant determinants of childhood weight and adiposity. J Pediatr 1985; 107: 104-7.

11. Baker DJ. Maternal nutrition, nutrition fetal, and disease in later life. Nutrition 1997; 13: 807-13.

12. Hall WG. The ontogeny of feeding in rats: ingestive and behavioral responses to oral infusions. J Comp Physiol Psychol 1979; 93: 977-1000.

13. Weller A, Gispan LH, Smith GP. Postingestive inhibitory controls of independent ingestion in 12-day-old rats. Physiol Behav 1996; 60: 361-4.
14. Hall WG, Rosenblatt JS. Development of nutritional control of food intake in suckling rat pups.Behav Biol 1978; 24: 413-27.

15. Hall WG, Cramer CP, Blass EM. Ontogeny of suckling in rats: transitions toward adult ingestion. J Comp Physiol Psychol 1977; 91: 1141-55.

16. Hall WG, Cramer CP, Blass EM. Developmental changes in suckling of rat pups. Nature $1975 ; 258$ : 318-20.

17. Alberts JR, Ronca AE. Fetal experience revealed by rats: psychobiological insights. Early Hum Dev 1993; 35: 153-6.

18. Pedersen PE, Williams CA, Blass EM. Activation and odor conditioning of suckling behavior in 3-day-old albino rats. J Exp Psychol Anim Behav Process 1982; 8: 329 41.

19. Klaus MH. Mother-child relationship: significant clinical observations. Katilolehti 1975; 80: 208-16.

20. Riul TR, Almeida PS, De-Oliveira LM, Almeida SS. Ethological analysis of mother-pup interactions and other behavioral reactions in rats: effect of malnutrition and tactile stimulation of the pups. Braz J Med Biol Res 1999 32: 975-83.

21. Butte NF, Wills C, Jean CA, Smith O, Garza C. Feeding patterns of exclusively breast-fed infants during the firs four months of life. Early Hum Dev 1985; 12: 291-300.

22. Halford JC, Blundell JE. Metergoline antagonizes fluoxetine-induced suppression of food intake but not changes in the behavioural satiety sequence. Pharmacol Biochem Behav 1996; 54: 745-51.

23. York DA. Peripheral and mechanisms regulating food intake and macronutrient selection. Obes Surg 1999; 9 471-9.

24. Lytle, LD. Control of eating behavior. In: Wurtman RJ, Wurtman JJ, editors. Nutrition and the brain. New York: Raven Press; 1977. p. 1-147.

25. Nagase H, Nakajuma A, Sekihara H, York DA, Bray GA. Regulation of feeding behavior, gastric emptying, and 
sympathetic nerve activity to interscapular brown adipose tissue by galanin and enterostatin: the involvement of vagal-central nervous system interactions. J Gastroenterol 2002; 37 Suppl 14: 118-27.

26. Rayner CK, Park HS, Doram SM, Chapman IM, Horowitz M. Effects of cholecystikinin on appetite and pyloric motility during physiological hyperglycemia. Am J Physiol Gastrointest Liver Physiol 2000: 278: 98-104.

27. De Castro JM, Balagura SA. Preprandial intake pattern in weanling rats ingesting a high fat diet. Physiol Behav 1976; 17: 401-5.

28. Weller A. The ontogeny of postingestive intake inhibition in rats. Appetite 2000; 34: 113

29. Hall WG, LaChaussee JL. Oral experience and the control of ingestion: lessons from development studies. Appetite 1993; 21: 305 .

30. Swithers SE, Miller GL, Hall WG. Habituation of oromotor responding to oral infusions in rat pups. Appetite 1991; 17: 55-67.

31. Levin R, Stern JM. Maternal influences on ontogeny of suckling and feeding rhythms in the rat. J Comp Physiol Psychol 1975; 89: 711-21.

32. Galef BG, Clark MM. Mother's milk and adult presence: two factors determining initial dietary selection by weaning rats. J Comp Physiol Phychol 1972; 78: 2202-25.

33. Galef BG, Clark MM. Parent-offspring interactions determine time and place of first ingestion of solid food by wild rat pups. Psychon Sci 1971; 25: 15-6.

34. OMS (Organização Mundial da Saúde). Proteção, promoção e apoio ao aleitamento materno: papel dos serviços materno-infantis. Genebra: A Organização; 1989.

35. Pozzo-Miller LD, Aoki A. Postnatal development of the hypothalamic ventro-medial nucleus: neurons and synapses. Cell Mol Neurobiol 1992; 12: 121-9.

36. Davidowa H, Plagemann A. Inhibition by insulin of hypothalamic VMN neurons in rats overweight due to postnatal overfeeding. Neuroreport 2001; 12: 3201-4.

37. Dietz WH. Critical periods in childhood for the development of obesity. Am J Clin Nutr 1994; 59: 955-9.

38. Alves JGB, Figueira F. Doenças do adulto com raízes na infância. Recife: Bagaço; 1998.

39. Plagemann A, Harder T, Rake A, Voits M, Fink H, Rohde W, Dorner G. Perinatal elevation do hypothalamic galaninergic neurons, and syndrome X-like alterations in adulthood of neonatally overfed rats. Brain Res 1999; 836: 146-55.

40. Davidowa H, Plagemann A. Decreased inhibition by leptin of hypothalamic arcuate neurons in neonatally overfed young rats. Neuroreport 2001; 11: 2795-98.

41. Yuan CS, Attele AS, Zhang L, Lynch JP, Xie JT, Shi ZQ. Leptin reduces body weight gain in neonatal rats. Pediatr Res 2000; 48: 380-3.
42. Davidowa H, Heidel E, Plagemann A. Differential involvement of dopamine D1 and D2 receptors and inhibition by dopamine of hypothalamic VMN neurons in early postnatally overfed juvenile rats. Nutr Neurosci 2002; 5: 27 36.

43. Meguid MM, Yang ZJ, Laviano A. Meal size and Sunumber: relationship to dopamine levels in the ventromedial hypothalamic nucleus. Am J Physiol Regul Integr Comp Physiol 1997; 272: R1925-30.

44. Hamosh M. Does infant nutrition affect adiposity and cholesterol levels in the adult? J Pediatr Gastroenterol Nutr 1988; 7: 10-6.

45. McGill HC, Mott GE, Lewis DS, McMahan CA, Jackson EM. Early determinants of adult metabolic regulation effects of infant nutrition on adult lipid and lipoprotein metabolism. Nutr Rev 1996; 54 Suppl 2: S31-40.

46. Owen CG, Whincup PH, Odoki K, Gilg JA, Cook DG. Infant feeding and blood cholesterol: a study in adolescents and a systematic review. Pediatrics 2002; 110: 597-608.

47. Noback CR, Eisenman LM. Some effects of protein-calorie undernutrition on the developing central nervous system of the rat. Anat Rec 1981; 201: 67-73.

48. Resnick O, Miller M, Forbes W, Hall R, Kemper T, Bronzino J, Morgane PJ. Developmental protein malnutrition influences on the central nervous system of the rat. Neurosci Biobehav Rev 1979; 3: 233-46.

49. Plagemann A, Harder T, Rake A, Melchior K, Rohde W, Dorner G. Hypothalamic nuclei are malformed in weanling offspring of low protein malnourished rat dams. J Nutr 2000; 130: 2582-90.

50. Souza SL. Ontogênese do comportamento alimentar em ratos e sua relação com a desnutrição [dissertação mestrado]. Recife: Departamento de Nutrição, Centro de Ciências da Saúde da Universidade Federal de Pernambuco; 2001

51. Barreto-Medeiros JM. Agressão intraespecífica e comportamento alimentar em ratos adultos: efeito da desnutrição pregressa e/ou de inibidor seletivo de recaptação da serotonina [dissertação mestrado]. Recife: Departamento de Nutrição, Centro de Ciências da Saúde da Universidade Federal de Pernambuco; 1998.

52. Barreto-Medeiros JM, Cabral Filho JE, De Souza SL, Freitas Silva SR, Mendes da Silva C, Deiró TCBJ, Monteiro JM, Guedes RCA, De Castro CMMB, Manhães de Castro R. Early malnourished rats are not affected by anorexia in duced by a seletive serotonin reuptake inhibitor in adult life. Nutr Neurosci 2002; 5: 211-4.

53. Forsdhahl A. Are poor living conditions in childhood and adolecence an important risk factor for arteriosclerotic heart disease? Br J Prev Soc Med 1997; 315: 837-40.

54. Fall CH, Vijayakumar M, Barker DJ, Osmond C, Duggleby S. Weight in infancy and prevalence of coronary heart disease in adult life. BMJ 1995; 310: 17-9.

Recebido em 27 de fevereiro de 2003

Versão final apresentada em 8 de julho de 2003

Aprovado em 2 de agosto de 2003 